\title{
Parásitos Zoonóticos en Ranas Destinadas a la Elaboración de Bebidas para el Consumo Humano en Lima, Perú
}

\author{
Zoonotic Parasites in Frogs Used for Preparing Beverages for Human \\ Consumption in Lima, Peru \\ Enrique Serrano-Martínez ${ }^{1,2}$, Marco Quispe H. ${ }^{1}$, Lucy Plascencia P. ${ }^{1}$, \\ Elizabeth Hinostroza M. ${ }^{1}$
}

\section{Resumen}

El presente estudio fue realizado para evaluar la contaminación por parásitos en ranas de la especie Telmatobius jelskii, empleadas en la preparación de extractos destinados al consumo humano directo en la forma de bebidas expendidas en áreas públicas. Se recolectaron 84 ranas en 21 establecimientos públicos de consumo de bebidas en el Cercado de Lima, Perú. Para la detección de parásitos gastrointestinales se realizaron técnicas coproparasitológicas directas y la técnica cualitativa de Travassos. Asimismo, para la detección de parásitos metazoos se revisó el intestino delgado y grueso, hígado, pulmón y vejiga urinaria. Todas las ranas presentaron parásitos de por los menos tres especies, identificándose la presencia de dos digeneos: Gorgoderina attenuata $(7.2 \%)$ y Gorgoderina sp (11.9\%), dos nematodos: Hedruris heyeri (100\%) y Capillaria sp (6.0\%), y dos protozoos: Cryptosporidium sp (100\%) y Balantidium sp (100\%), ambos de carácter zoonótico. Los resultados demuestran una elevada contaminación por parásitos zoonóticos en las ranas empleadas en la elaboración de extractos, lo cual implica un riesgo de infección de los consumidores de extracto de rana, toda vez que los extractos son elaborados con ranas enteras que no son sometidas a proceso térmico.

Palabras clave: salud pública; Gorgoderina attenuata; cryptosporidiosis; zoonosis; bebida

\section{Abstract}

This study was conducted to evaluate the contamination for parasites in the frog Telmatobius jelskii, used in the preparation of extracts intended for direct human consumption in the form of beverages expended in public areas. Frogs $(n=84)$ were taken

\footnotetext{
${ }^{1}$ Grupo SALUVET-UPCH, Facultad de Medicina Veterinaria y Zootecnia, Universidad Peruana Cayetano Heredia, Lima, Perú

${ }^{2}$ E-mail: enrique.serrano@upch.pe
}

Recibido: 2 de julio de 2016

Aceptado para publicación: 15 de marzo de 2016 
in 21 food carts in Lime city center. Direct coproparasitological techniques and the Travassos technique were used for the detection of gastrointestinal parasites. For the detection of metazoan parasites the small and large intestine, liver, lung and bladder were revised. All frogs had parasites of at least three species, identifying the presence of two digenea: Gorgoderina attenuata (7.2\%) and Gorgoderina sp (11.9\%), two nematodes: Hedruris heyeri (100\%) and Capillaria sp (6.0\%) and two zoonotic protozoa: Cryptosporidium sp (100\%) and Balantidium sp (100\%). The results showed high contamination with parasites in frogs that are used in the preparation of extracts, which implies a risk of infection for consumers if considering that extracts were prepared with whole frogs that are not are not subjected to cooking processes.

Key words: parasites; frogs; Gorgoderina attenuata; Cryptosporidium sp; zoonotic; beverages

\section{INTRODUCCIÓN}

Los anfibios cumplen un rol fundamental en la mega biodiversidad del Perú y su población es importante para el equilibrio del ecosistema (MINAM, 2015). En 2008 se reportó la presencia de 538 especies de anfibios (Aguilar et al., 2010), número que se ha incrementado en los últimos años con nuevos descubrimientos; no obstante, esta biodiversidad ha disminuido a nivel mundial, principalmente por la pérdida de sus hábitats, enfermedades micóticas (quitridiomicosis) y sobreexplotación (Young et al., 2004).

Los anfibios del género Telmatobius y Atelopus se encuentran en el país en la categoría de amenaza y casi amenazado, respectivamente (Barrera, 2004; von May et al., 2008). Estos anfibios habitan mayormente en arroyos y lagos entre los 2000 y $3000 \mathrm{msnm}$ (Pérez, 2005), son excelentes indicadores de la calidad del hábitat terrestre o acuático y su reproducción ocurre mayormente en épocas de lluvias (Franco y Jara, 2005).

En el género Telmatobius, los más frecuentes son T. culeus y T. jelskii (Young et al, 2004; Pérez, 2005), poblaciones que han disminuido por diferentes causas, incluyendo su empleo en jugos exóticos (extracto de rana), ya que es considerado como un remedio natural para la bronquitis, aumento de la lucidez y la virilidad. Asimismo, otras especies son criadas con fines comerciales por su carne y para la preparación de embutidos (Ramos et al., 2000; von May et al., 2008). Teniendo en cuenta lo anterior, existe la posibilidad de que estos anuros puedan representar un riesgo para la salud pública. Se han reportado casos de salmonelosis por el consumo de extracto de ranas en los mercados (Barrera, 2004). Por otro lado, es conocido que, a nivel mundial, los anuros terrestres están más parasitados por digeneos o nematodos que los anfibios acuáticos (Iannacone, 2003). En el caso de preparación de bebidas (extractos) en el Cercado de Lima, estos son elaborados con ranas enteras, no evisceradas, y que no son sometidas a proceso térmico alguno.

El presente estudio tuvo por objetivo identificar las especies de parásitos presentes en ranas empleadas en el Cercado de Lima para la elaboración de bebidas para el consumo humano, así como determinar las frecuencias de infección, especialmente de aquellos agentes de importancia zoonótica.

\section{Materiales y Métodos}

El muestreo de ranas se llevó a cabo en los establecimientos públicos de venta de jugos frescos y de consumo de bebidas (ex- 
tractos) ubicados en el Cercado de Lima, Perú. Entre ellos, los ubicados en la Av. Abancay, Av. Ayacucho, Av. Puno, Av. Ancash, Av. Venezuela, Av. Tacna y Av. Grau. En general, se adquirieron cuatro ranas por cada puesto. El análisis de laboratorio se realizó en el Laboratorio de Parasitología Animal de la Facultad de Medicina Veterinaria y Zootecnia de la Universidad Peruana Cayetano Heredia, Lima.

Entre junio de 2014 y enero de 2015 se recolectaron 84 ranas muertas (23 machos y 61 hembras) de 21 puestos de bebidas, las cuales estaban destinadas al consumo humano. Los animales fueron extraídos de sus recipientes y fueron colocados en cajas térmicas para su traslado al laboratorio. El sexo de los anuros Telmatobius jelski fue determinado durante la necropsia de los animales, examinado las gónadas en la cavidad corporal. La identificación de la especie fue confirmada siguiendo los lineamientos de Franco et al. (1999).

En la necropsia se retiraron los tractos gastrointestinales, los cuales fueron procesados mediante la técnica cualitativa de Travassos (Leguía y Casas, 1999). Asimismo, se revisaron las mucosas de los órganos, ya lavados, con el fin de detectar a aquellos helmintos adheridos a la mucosa o por debajo de ella.

Los helmintos fueron fijados con alcohol etílico al $70 \%$ o formol al $10 \%$, coloreados y montados, siguiendo las recomendaciones de Eiras et al. (2000). Los nematodos se clarificaron en una mezcla de alcohol con fenol para su identificación, en tanto que los platelmintos se lavaron y fijaron en formol al $10 \%$, previo aplanamiento entre lámina y laminilla por 24 horas, para finalmente colorearlos con carmín acético de Semichon, deshidratarlos, clarificarlos y montarlos en preparaciones permanentes con Bálsamo de Canadá (Salgado, 2009). Adicionalmente, las heces se evaluaron por observación directa en solución salina fisiológica o con lugol parasitológico con el fin de observar la presencia de huevos. En la búsqueda de metazoos parásitos se incluyó el intestino delgado y grueso, hígado, pulmón y vejiga urinaria. Para la determinación taxonómica de los helmintos parásitos se usaron los criterios establecidos por Khalil et al. (1994).

La evaluación parasitaria de Cryptosporidium sp se realizó mediante la coloración Zeihl-Neelsen y de los demás parásitos por observación directa. Los valores de prevalencia, intensidad media de infección y abundancia media se calcularon de acuerdo al criterio de Bush et al. (1997).

\section{Resultados}

Todas las ranas recolectadas fueron de la especie Telmatobius jelski. Se identificaron parásitos digeneos (Gorgoderina attenuata y Gorgoderina $s p$ ) en vejiga urinaria y riñón, nematodos (Hedruris heyeri y Capillaria sp) en estómago e intestinos y dos protozoos (Cryptosporidium sp y Balantidium $\mathrm{sp}$ ) en intestino, estos últimos de carácter zoonótico (Cuadros 1 y 2, Figura 1). La mayor intensidad media (IM) y abundancia media (AM), no significativa, se presentó en hembras frente a los machos (Cuadro 2).

Se observaron infecciones con tres especies de parásitos en las 84 ranas y con $4 \mathrm{y}$ 5 especies por rana en el 21.4 y $3.6 \%$, respectivamente. Los parásitos hallados se muestran en el Cuadro 1.

En el caso de Gorgoderina attenuata, se encontró asociación estadística (Chi cuadrado) entre la infección con este parásito y el tamaño de las ranas $(<60 \mathrm{~mm} v s \geq 60 \mathrm{~mm}$ de longitud; $p<0.05$ ), pero no la hubo con el sexo de la rana o su procedencia. Por otro lado, no hubo asociación estadística entre helmintos de interés zoonótico y otras variables en estudio. 
Cuadro 1. Frecuencia y ubicación de parásitos en ranas Telmatobius jelskii $(\mathrm{n}=84)$, procedentes de establecimientos comercializadores de bebidas (extractos) destinados al consumo humano, en el Cercado de Lima, Perú

\begin{tabular}{|c|c|c|c|}
\hline Parásito & Positivos $(\%)$ & Lugar de ubicación & $\begin{array}{l}\text { Carácter } \\
\text { zoonótico }\end{array}$ \\
\hline \multicolumn{4}{|l|}{ Digeneo } \\
\hline Gorgoderina attenuata & 7.1 & $\begin{array}{l}\text { Vejiga urinaria, } \\
\text { riñones }\end{array}$ & No \\
\hline Gorgoderina $\mathrm{sp}$ & 11.9 & $\begin{array}{l}\text { Vejiga urinaria, } \\
\text { riñones }\end{array}$ & No \\
\hline \multicolumn{4}{|l|}{ Nematodo } \\
\hline Hedruris heyeri & 100.0 & $\begin{array}{l}\text { Estómago e } \\
\text { Intestinos }\end{array}$ & No \\
\hline Capillaria $\mathrm{sp}$ & 6.0 & Intestino delgado & No \\
\hline \multicolumn{4}{|l|}{ Protozoo } \\
\hline Cryptosporidium $\mathrm{sp}$ & 100.0 & Intestinos & Sí \\
\hline Balantidium sp & 100.0 & Intestinos & Sí \\
\hline
\end{tabular}

Cuadro 2. Frecuencia (P), Intensidad Media (IM) y Abundancia Media (AM) de parásitos en ranas Telmatobius jelskii, procedentes de establecimientos comercializadores de bebidas (extractos) destinados al consumo humano, en el Cercado de Lima, Perú

\begin{tabular}{lcccccc}
\hline \multirow{2}{*}{ Parásito } & \multicolumn{3}{c}{ Macho } & \multicolumn{3}{c}{ Hembra } \\
\cline { 2 - 7 } & $\begin{array}{c}\mathrm{P}(\%) \\
(\mathrm{n}=23)\end{array}$ & $\mathrm{IM}^{1}$ & $\begin{array}{c}\mathrm{AM} \\
(\mathrm{n}=23)\end{array}$ & $\begin{array}{c}\mathrm{P}(\%) \\
(\mathrm{n}=61)\end{array}$ & $\mathrm{IM}^{1}$ & $\begin{array}{c}\mathrm{AM} \\
(\mathrm{n}=61)\end{array}$ \\
\hline Gorgoderina attenuata & 8.7 & $1.5(2)$ & 0.13 & 6.6 & $1.8(4)$ & 0.11 \\
Gorgoderina sp & 13.0 & $1.7(3)$ & 0.22 & 11.5 & $1.6(7)$ & 0.18 \\
Hedruris heyeri & 100.0 & $51.4(23)$ & 51.43 & 100.0 & $55.9(61)$ & 55.93 \\
Capillaria sp & 8.7 & $2.0(2)$ & 0.17 & 4.9 & $2.3(3)$ & 0.11 \\
Cryptosporidium $\mathrm{sp}$ & 100.0 & $90.9(23)$ & 90.91 & 100.0 & $92.1(61)$ & 92.08 \\
Balantidium $\mathrm{sp}$ & 100.0 & $118.4(23)$ & 118.35 & 100.0 & $193.4(61)$ & 193.41 \\
\hline
\end{tabular}

${ }^{1}$ Número de parásitos entre paréntesis 




Figura 1. Parásitos gastrointestinales observados en ranas Telmatobius jelskii procedentes de 21 establecimientos comercializadores de bebidas (extractos) de consumo humano, en el Cercado de Lima. Perú. A) Hedruris sp (10x); B) Parte anterior Hedruris heyeri (10x); C) Parte posterior de Hedruris heyeri (40x); D) Gorgoderina $\mathrm{sp}$ (10x); E) G. attenuata (10x); F) Parte anterior de G. attenuata (40x); G) Parte posterior de G. attenuata (40x); H) Cryptosporidium sp (100x); I) Balantidium sp $(40 \mathrm{x})$

\section{Discusión}

Todas las ranas se encontraban parasitadas, principalmente por el nematodo Hedruris heyeri en estómago e intestinos y los protozoos Cryptosporidium sp y Balantidium sp en el intestino. El 100\% de ranas $T$. jelskii positivas a parásitos fue mayor que el $41.8 \%$ reportado en el trabajo de Iannacone (2003). Otros estudios realizados en $T$. jelskii han registrado la presencia de los nematodos Aplectana hylambatis (Iannacone, 2003), Camallanus sp, Falcaustra condorcanqui (Ibáñez y Córdova, 1976) y Hedruris sp (Tantaleán et al., 1992, Sarmiento et al., 1999).
En relación a la presencia de digeneos y cestodos, el estudio realizado por Iannacone (2003) en 67 ranas de la especie T. jelskii, reportó la presencia del digeneo Gorgoderina parvicava (40.3\%) y del cestodo Cylindrotaenia americana (3\%). Gorgoderina sp tiene distribución mundial, especialmente en zonas neotropicales, pero se desconoce el comportamiento parasitario; sin embargo, parece tener un ciclo biológico similar a la Fasciola puesto que ambos necesitan del agua y bivalvos para desarrollarse. A nivel mundial, existen 51 especies de Gorgoderina y en el Perú se han registrado en siete especies de anfibios dentro de tres familias - Telmatobiidae, Leptodactylidae y buffonidae - (Chero et al., 2014). Asimismo, 
estudios realizados en ranas toros (Lithobates catesbeianus) y ranas leopardos (Lithobates pipiens) de Norteamérica, encontraron que Gorgoderina sp tiene predilección por órganos específicos, tales como la vejiga urinaria (sapos leopardos) y riñón (ranas toros), respectivamente (Bolek et al., 2009).

De los parásitos hallados, Cryptosporidium sp y Balantidium sp resaltan por su importancia zoonótica. Según Xiao (2010), el protozoario Cryptosporidium sp es cosmopolita y de importancia no solo en salud pública, sino en animales, por causar severos cuadros gastrointestinales. La dosis infectiva para producir una infección en humanos es de 132 ooquistes (Garza y Morales, 2002; Parte-Pérez et al., 2005). Por otro lado, Balantidium sp tiene como reservorio habitual al humano y los cerdos, pero también a los roedores y carnívoros (Nakauchi, 1998; Vásquez y Vidal, 1999; Schuster y Ramirez, 2008), artiodáctilos y aves (Ponce et al., 2011), así como en peces y anfibios (Uribarren, 2014). La balantidiosis en el hombre es poco frecuente, pudiendo llegar al 10\% de portadores asintomáticos (Vásquezy Vidal, 1999). La infección se presenta al tener contacto con cerdos infectados o al existir deficientes condiciones de higiene ambiental. Los trofozoites se alojan en el lumen del intestino grueso, invadiendo las criptas intestinales, donde producen cuadros de disentería (Shuster et al., 2008).

\section{Conclusiones}

- $\quad$ El $100 \%$ de ranas Telmatobius jelskii destinadas al consumo humano mediante su utilización en la preparación de bebidas (extractos) en puestos de venta en el Cercado de Lima, Perú, estuvieron infectados con algún tipo de forma parasitaria.

- Se identificaron dos especies de trematodos, dos especies de nematodos $\mathrm{y}$ dos especies de protozoos.
- Cryptosporidium sp y Balanditidium sp fueron los agentes parasitarios de importancia zoonótica hallados en ranas $T$. jelskii.

\section{Literatura Citada}

1. Aguilar C, Ramírez C, Rivera D, SiuTing K, Suarez J, Torres C. 2010. Anfibios andinos del Perú fuera de áreas naturales amenazas y estado de conservación. Rev Per Biolo 17: 5-28. doi: 10.15381/rpb.v17i1.46

2. Barrera S. 2004. Enfermedades infecciosas y su papel en la declinación mundial de las poblaciones de anfibios. CONABIO Biodiversitas 56: 1-6.

3. Bolek M, Snyder S, Janovy J. 2009. Redescription of the frog bladder fluke Gorgoderina attenuate from the Northern leopard frog, Rana pipiens. $\mathrm{J}$ Parasitol 95. [Internet]. Disponible en: http://www.matthewbolek.com/ publications/mattspapers/ Gorgoderina\%20attenuata\%20redescription.pdf

4. Bush A, Lafferty K, Lotz J, Shostak A. 1997. Parasitology meets ecology on its own terms: Margolis et al., revisited. J Parasitol 83: 575-583. doi: 10.2307/ 3284227

5. Chero J, Cruces C, Iannacone J, Sáenz G, Alvariño L, Luque J, Morales V. 2014. Parásitos gastrointestinales en tres especies de Telmatobius (Anura: Telmatobiidae) de la zona alto andina, Perú. Neotropical Helminthol 8: 439-461.

6. Eiras J, Takemoto R, Pavanelli G. 2000. Métodos de estudo e técnicas laboratoriais em parasitología de peixes. Brasil: Universidadde Estadual de Maringa. $171 \mathrm{p}$.

7. Franco J, Jara M. 2005. Los anfibios y reptiles del valle del Cusco. En: Historia natural del valle del Cusco. Sociedad Protectora de la Naturaleza, Cusco. p 339-364. 
8. Garza V, Morales M. 2002. Agua y salud: Cryptosporidium parvum, agente causal de una nueva enfermedad relacionada con el agua. Rev Fac Salud Pública Nutr 3(1). [Internet]. Disponible en: http://www.respyn.uanl.mx/iii/1/ensayos/ agua_salud.html

9. Iannacone J. 2003. Helmintos parásitos de Telmatobius jelskii (Peters) (Anura, Leptodactylidae) de Lima, Perú. Rev Bras Zool 20: 131-134. doi: 10.1590/ S0101-81752003000100016

10. Ibáñez N, Córdova E. 1976. Quatro especies nuevas de nematodos del sur del Perú y redescripción de Hedruris orestiae Moniez, 1889. Mem Inst Oswaldo Cruz 74: 231-254. doi: 10.1590/ S0074-02761976000300004.

11. Khalil L, Jones A, Bray R. 1994. Keys to the cestode parasites of vertebrates. Wallingford, Oxfordshire, UK: CAB International. $768 \mathrm{p}$.

12. Leguía G, Casas E. 1999. Enfermedades parasitarias y atlas parasitológico de camélidos sudamericanos. Lima, Perú: Ed. De Mar. 190 p.

13. [MINAM] Ministerio del Ambiente. 2015. Guía de inventario de la fauna silvestre. 83 p. [Internet]. Disponible en: http://www.minam.gob.pe/patrimonionatural/wp-content/uploads/sites/6/2013/ $10 /$ G U \% C $3 \% 83$ - A - d e - fauna silvestre.compressed.pdf

14. Nakauchi K. 1998. The prevalence of Balantidium coli infection in fifty-six mammalian species. Vet Med Sci 61: 6365. doi: $10.1292 /$ jvms. 61.63

15. Parte-Pérez M, Bruzual E, Brito A, Hurtado M. 2005. Cryptosporidium spp y criptosporidiosis. Rev Soc Ven Microbiol 25(1):

16. Pérez M. 2005. Cría en cautividad y uso sostenible de la rana gigante del lago Titicaca (Telmatobius culeus). Monogr Herpetol 7: 261-271.

17. Ponce-Gordo F, Fonseca-Salamanca F, Martínez-Díaz R. 2011. Genetic heterogeneity in internal transcribed spacer genes of Balantidium coli (Litostomatea, Ciliophora). Protist 162: 774-794. doi: 10.1016/j.protis.2011.06.008

18. Ramos L, Gallegos N, Quispe L. 2000. Evaluación de la información disponible del suri, pisaca y rana gigante del lago. Puno: Universidad Nacional del Altiplano. $109 \mathrm{p}$.

19. Schuster F, Ramirez L. 2008. Current world status of Balantidium coli. American Society for Microbiology. Clin Microbiol Rev 21: 626-638.

20. Salgado G. 2009. Manual de práctica de parasitología con énfasis en helmintos parásitos de peces agua dulce y otros animales silvestres de México. México: Instituto de Biología, UNAM. 56 p. [Internet]. Disponible en: http:// www.ibiologia.unam.mx/pdf/directorio/s/ salgado/manual/manual_prac_parasitol.pdf

21. Sarmiento L, Tantaleán M, Huiza A. 1999. Nematodos parásitos del hombre y de los animales en el Perú. Rev Peru Parasitol 14: 9-65.

22. Tantaleán M, Sarmiento L, Huiza A. 1992. Digeneos (Trematoda) del Perú. Bol Lima 80: 47-84.

23. Uribarren T. 2014. Balantidiosis o balantidiasis. Departamento de Microbiología y Parasitología. Universidad Nacional Autónoma de México. 4 p. [Internet]. Disponible en: http://www.facmed.unam.mx/-deptos/microbiologia/ parasitologia/balantidiasis.html

24. Vásquez W, Vidal J. 1999. Colitis balantidiásica: a propósito de un caso fatal en el departamento de Huancavelica. Anales Fac Med 60: 119-123. doi: 10.15381/anales.v60i2.4480

25. von May R, Catenazzi A, Angulo A, Brown JL, Carrillo J, Chávez G, et al. 2008. Current state of conservation knowledge on threatened amphibian species in Peru. Trop Conserv Sci 1: 376396. 
26. Xiao L. 2010. Molecular epidemiology of cryptosporidiosis: an update. Exp Parasitol 124: 80-89. doi: 10.1016/ j.exppara.2009.03.018
27. Young BE, Suart SN, Chanson JS, Cox NA, Boucher TM. 2004. Disappearing jewels: the status of new world amphibians. Arlington, Virginia, USA: Nature Serve. 60 p. 